Mon. Not. R. Astron. Soc. 000, 000-000 (0000) Printed 21 March $2022 \quad$ (MN LATEX style file v2.2)

\title{
Collision strengths and transition probabilities for Co II infrared forbidden lines
}

\author{
P.J. Storey ${ }^{1 \star}$, C.J. Zeippen ${ }^{2}$, Taha Sochi $^{1}$ \\ ${ }^{1}$ Department of Physics and Astronomy, University College London, Gower Street, London WC1E 6BT, UK \\ ${ }^{2}$ LERMA, Observatoire de Paris, ENS, UPMC, UCP, CNRS, 5 Place Jules Janssen, F-92195 Meudon Cedex, France
}

Accepted 2015 November 24. Received 2015 November 24; in original form 2015 September 10

\begin{abstract}
We calculate collision strengths and their thermally-averaged Maxwellian values for electron excitation and de-excitation between the fifteen lowest levels of singly-ionised cobalt, $\mathrm{Co}^{+}$, which give rise to emission lines in the near- and mid-infrared. Transition probabilities are also calculated and relative line intensities predicted for conditions typical of supernova ejecta. The diagnostic potential of the 10.52, 15.46 and $14.74 \mu \mathrm{m}$ transition lines is briefly discussed.
\end{abstract}

Key words: atomic data - atomic processes - radiation mechanisms: non-thermal supernovae: general - infrared: general.

\section{INTRODUCTION}

Cobalt transition lines are useful astronomical probes especially in analysing the observational data of supernovae (SNe) where these emissions can be used to assess the nucleosynthesis and decay processes associated with the SN explosion. The spectra of supernova ejecta show prominent infrared forbidden lines of singly- and doubly-ionised ions of the iron group elements: nickel, cobalt and iron, where the formation of these elements is largely based on the radioactive decay chain ${ }^{56} \mathrm{Ni} \rightarrow{ }^{56} \mathrm{Co} \rightarrow{ }^{56} \mathrm{Fe}$ (Colgate \& McKee 1969, Kuchner et al 1994, Bowers et al 1997, Liu et al 1997. Churazov et al 2014). Hence, the lines of these elements can be used to examine the underlying nuclear processes. These lines can also be used for the determination of particle number density and for analysing the thermodynamic conditions of these objects (Nussbaumer \& Storey 1988, Kuchner et al 1994, Bowers et al 1997, Adelman et al| 2000, del Peloso et al 2005 Bergemann et al 2010).

Observations of cobalt lines in super and symbiotic novae, largely forbidden transitions in the infrared and optical parts of the spectrum, have been reported in a number of studies (Axelrod 1980 Li et al 1993, Jennings et al 1993. Kuchner et al 1994, Dinerstein 1995: Bowers et al 1997| Liu et al 1997. McKenna et al 1997 |Churazov et al 2014). Definite or tentative observations of certain forbidden transitions of Co in the spectra of planetary nebulae and H II regions have also been reported (Baluteau et al 1995. Sharpee et al| 2003. Esteban et al 2004 |Zhang et al 2005. Pottasch \& Surendiranath 2005. Sharpee et al 2007; Wang \& Liu 2007.

* E-mail: pjs@star.ucl.ac.uk
Fang \& Liu 2011). Cobalt lines have also been observed in other astronomical objects like early-type stars Adelman et al 2000) and cool stars (Bergemann et al 2010) as well as in the solar spectrum (Kerola \& Aller $|1976|$ Salih et al 1985. Pickering et al 1998, Bergemann et al 2010). Relevant observational data related to the $\mathrm{SN}$ spectra in general and to the IR lines in particular have been gathered in the past using various instruments such as the Infrared Spectrograph (IRS) on the Spitzer Space Telescope and Himalaya Faint Object Spectrograph Camera (HFOSC) on the Himalayan Chandra Telescope (see e.g. Sahu et al (2006) and Jerkstrand et al (2012).

There are several experimental investigations (see e.g. Sugar \& Corliss (1981, 1985); Salih et al (1985); Crespo López-Urrutia et al (1994); Mullman et al (1998a b); Pickering et al (1998); Ruffon \& Pickering (2013)) in which atomic data, such as energy levels and radiative transition probabilities, have been reported.

Nussbaumer \& Storey (1988) reported calculations of forbidden transition probabilities between the energetically lowest eight levels of $\mathrm{Co}^{+}$using a multi-configuration atomic model. The calculations, which are based on employing the Superstructure code (Eissner et al 1974, Nussbaumer \& Storey 1978), have also included summarisation of similar calculations for Co I and Co III transitions. These data were intended to facilitate the analysis of the observations of SNe in general and SN1987A in particular. Raassen et al (1998) conducted experimentally-based calculations of oscillator strengths and transition probabilities for a number of dipole-allowed and dipole-forbidden transitions of singlyionised cobalt using a semi-empirical approach based on employing an orthogonal operator method within an inter- 
mediate coupling scheme. The data were intended for use in analysing astronomical spectra from such objects as Co stars and late type supernovae. For similar purposes, Quinet (1998) computed radiative transition probabilities for the dipole-forbidden transitions between the 47 metastable energy levels in the $3 \mathrm{~d}^{8}, 3 \mathrm{~d}^{7} 4 \mathrm{~s}$ and $3 \mathrm{~d}^{6} 4 \mathrm{~s}^{2}$ configurations of the singly-ionised cobalt using a configuration interaction Hartree-Fock relativistic approach with optimised radial parameters based on the available observed energy levels.

In this paper, we report computational atomic data in the form of collision strengths and their thermally-averaged Maxwellian values for electron excitation and de-excitation between the 15 lowest levels of $\mathrm{Co}^{+}$as well as transition probabilities for a number of the forbidden lines of Co II in the infrared section of the spectrum. The investigation is generally based on employing the R-matrix method and codes $^{1}$ (Berrington et al 1974, 1987, Hummer et al 1993. Berrington et al 1995) where the scattering calculations are conducted using a 13-configuration atomic target within a Breit-Pauli intermediate coupling approximation.

In Section 2 of the present paper, we discuss the target used in our $\mathrm{Co}^{+}$model and give the resulting transition probabilities. Details of the Breit-Pauli R-matrix electron scattering calculations can be found in Section 3 . Results and some diagnostics appear in Section 4 . The paper is finalised in Section 5 with general conclusions and discussion.

\section{$2 \mathrm{Co}^{+}$ATOMIC STRUCTURE}

\subsection{The scattering target}

A schematic diagram of the term structure of Co II is shown in Figure 1. The lowest 19 terms are of even parity from the configurations $3 \mathrm{~d}^{8}$ and $3 \mathrm{~d}^{7} 4 \mathrm{~s}$. In this work we are concerned with the excitation mechanisms of the forbidden transitions among these even-parity terms, particularly those lying in the near- and mid-infrared. The levels giving rise to midinfrared transitions and the corresponding wavelengths are shown in Figure 2. Transitions from higher terms give rise to near-infrared lines but these are expected to be weaker at the temperatures typical of supernova ejecta.

It is expected that the odd-parity terms of the $3 \mathrm{~d}^{7} 4 \mathrm{p}$ configuration will give rise to resonance series that affect the collision strengths for excitation of the low-lying even-parity levels and should therefore be included in the target for the electron scattering calculation. The extent of our target is shown by the solid line in Figure 1 and includes 26 terms and 72 levels. The target states were expanded over the set of thirteen electron configurations listed in Table 1 and the target wavefunctions were calculated with the program AUTOSTRUCTURE, (Eissner et al 1974, Nussbaumer \& Storey 1978, Badnell 2011), which uses radial wavefunctions calculated in scaled Thomas-Fermi-Dirac statistical model potentials. The scaling parameters were determined by minimising the sum of the energies of all the target terms, computed in $L S$-coupling, i.e. neglecting all relativistic effects. The resulting scaling parameters are given in Table 2 where

1 See Badnell: R-matrix write-up on WWW. URL: amdpp.phys.strath.ac.uk/UK_RmaX/codes/serial/WRITEUP.

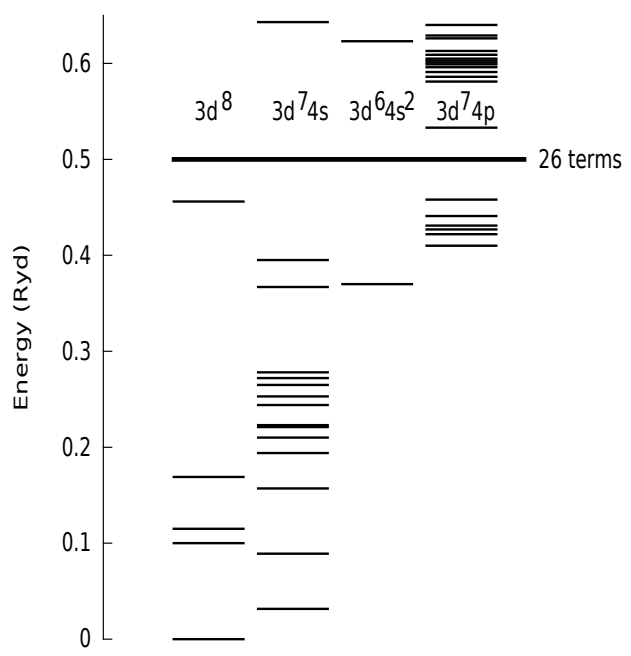

Figure 1. Schematic term energy diagram of Co II. The solid line shows the extent of the close-coupled target states.

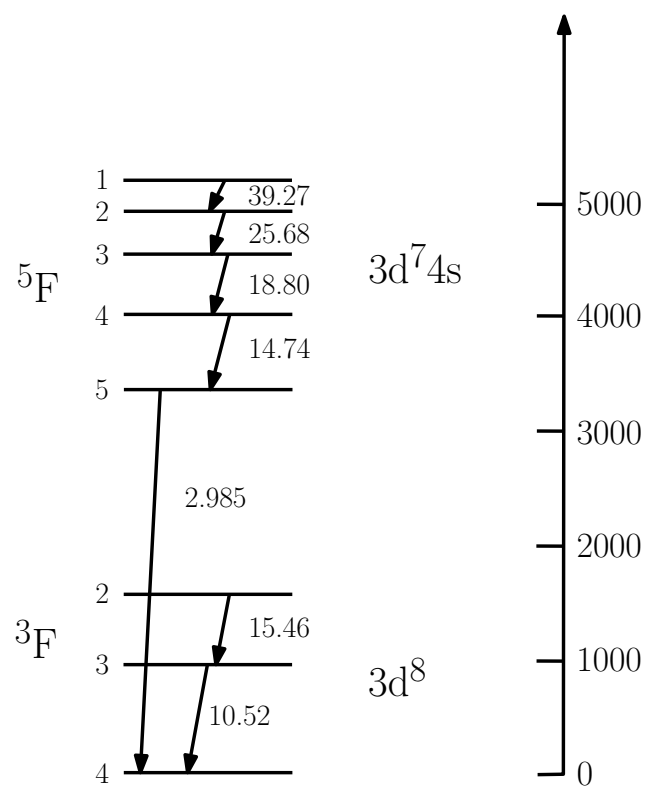

Figure 2. The lowest levels of $\mathrm{Co}^{+}$and the mid-infrared transitions between them. The energy scale on the right is in $\mathrm{cm}^{-1}$ while the transition wavelengths next to the arrows are in $\mu \mathrm{m}$.

a negative scaling parameter, $\lambda_{n l}$, signifies a hydrogenic correlation orbital with nuclear charge $27\left|\lambda_{n l}\right|$.

In Table 3 we compare the term energies calculated with our target with experiment for the 26 terms of the target. The calculated term energies include one-body relativistic shifts, the mass and Darwin terms, and the spin-orbit interaction. This is the level of approximation that applies in the R-matrix code used for the electron scattering calculation. In Table 4 we compare the calculated energies of the 15 lowest levels with experimental values. In this table we also show the results obtained when the two-body fine structure interactions described by Eissner et al (1974) are included. The overall fine-structure splittings of the tabulated terms are significantly improved when the two-body terms are in- 
Table 1. The target configuration basis where the core structure is suppressed. The bar indicates a correlation orbital.

\begin{tabular}{ll}
$3 d^{8}$ & $3 d^{7} 4 p$ \\
$3 d^{7} 4 s$ & $3 d^{6} 4 s 4 p$ \\
$3 d^{6} 4 s^{2}$ & \\
$3 d^{6} 4 p^{2}$ & \\
$3 d^{7} \overline{4} d$ & \\
$3 d^{7} \overline{5} s$ & \\
$3 d^{7} \overline{5} d$ & \\
$3 d^{7} \overline{6} d$ & \\
$3 d^{6} 4 s \overline{4} d$ & \\
$3 d^{6} \overline{4} d^{2}$ & \\
$3 d^{5} 4 s^{2} \overline{4} d$ & \\
\hline
\end{tabular}

Table 2. Potential scaling parameters. The bar over the principal quantum number and the minus sign attached to the value of a scaling parameter signifies a correlation orbital.

\begin{tabular}{rrrrrr}
\hline $1 \mathrm{~s}$ & 1.43187 & $2 \mathrm{p}$ & 1.08106 & $3 \mathrm{~d}$ & 1.02832 \\
$2 \mathrm{~s}$ & 1.13734 & $3 \mathrm{p}$ & 1.04220 & $\overline{4} \mathrm{~d}$ & -0.34647 \\
$3 \mathrm{~s}$ & 1.05922 & $4 \mathrm{p}$ & 0.98776 & $\overline{5} \mathrm{~d}$ & -2.19279 \\
$4 \mathrm{~s}$ & 0.98959 & & & $\overline{6} \mathrm{~d}$ & -2.00375 \\
$\overline{5} \mathrm{~s}$ & -0.55000 & & & & \\
\hline
\end{tabular}

cluded, with the average difference from experiment falling from $5.3 \%$ to $1.8 \%$. Table 4 serves as a key to the levels for use in later results for transition probabilities, collision strengths and effective collision strengths.

A further measure of the quality of the target is a comparison between weighted oscillator strengths, $g f$, calculated in the length and velocity formulations. Good agreement between the two formulations is a necessary but not sufficient condition for ensuring the quality of the target wavefunctions. This comparison, given in Table 5 , shows an average difference in the absolute values of $g f$ of $10.9 \%$ between the two formulations, which we consider acceptable for an open d-shell system.

\subsection{Transition probabilities}

Using the target wavefunctions described above, we computed the forbidden transition probabilities among the lowlying even parity terms. The calculated energies are replaced by experimental energies to correct the energy factors connecting the $a b$ initio calculated line strengths to the transition probabilities. The results are given in Table 6 with comparison to values obtained from previous investigations. We only tabulate those probabilities from a given upper level which exceed $1 \%$ of the total probability from that level. We also tabulate the magnetic dipole transition probabilities between the levels of the ${ }^{3} \mathrm{~F}$ and ${ }^{5} \mathrm{~F}$ terms calculated assuming the states are described by pure LS-coupling, in which case the line strength is given by a simple formula (Nussbaumer \& Storey 1988).

The mid-infrared transitions between the levels of the $3 \mathrm{~d}^{8} \quad a^{3} \mathrm{~F}$ and $3 \mathrm{~d}^{7} 4 \mathrm{~s} \quad a^{5} \mathrm{~F}$ terms are dominated by magnetic dipole decays which change $J$ by unity, with electric quadrupole transitions being orders of magnitude smaller. This leads to a step-wise decay through the levels within each term, as illustrated in Figure 2 The lowest $a^{5} \mathrm{~F}$ level,
Table 3. Energies of the 26 target terms in $\mathrm{cm}^{-1}$. The calculated values include only the spin-orbit contribution to the finestructure energies. Core structure is suppressed from all configurations.

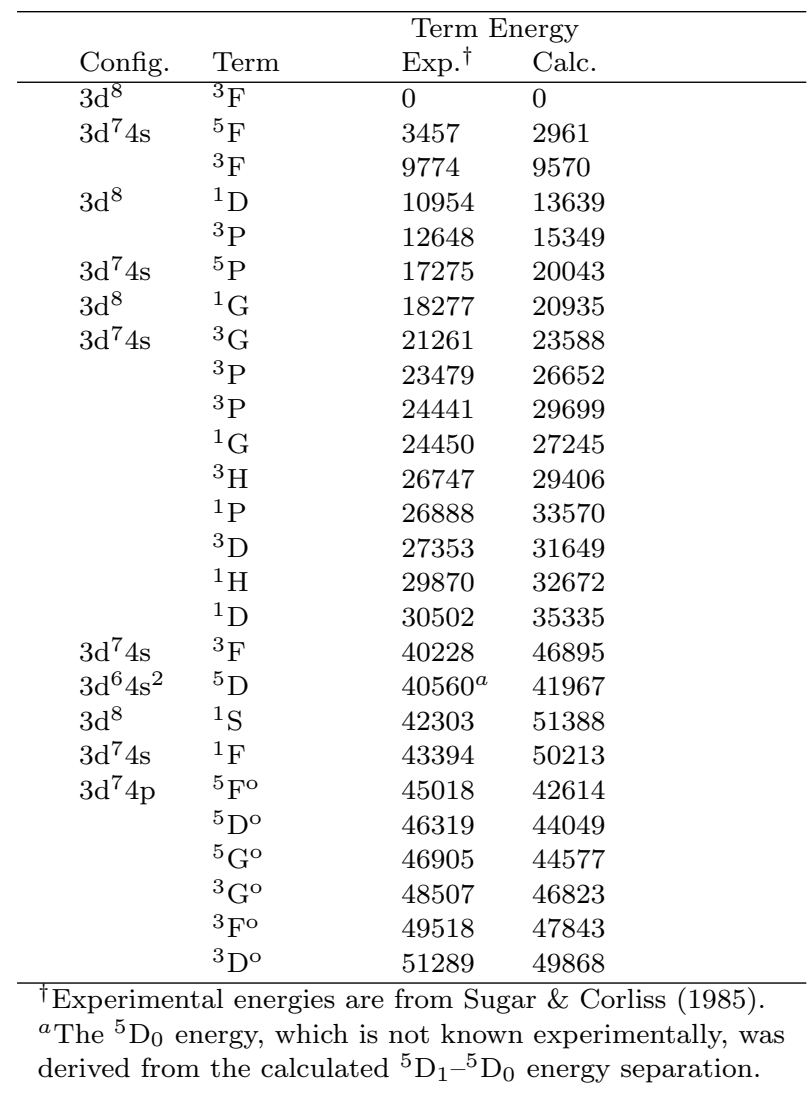

Table 4. Energies in $\mathrm{cm}^{-1}$ of the 15 lowest target levels.

\begin{tabular}{|c|c|c|c|c|c|}
\hline Index & Config. & Level & Exp. $^{1}$ & Calc. $^{2}$ & Calc. $^{3}$ \\
\hline 1 & $3 \mathrm{~d}^{8}$ & $a^{3} \mathrm{~F}_{4}$ & 0. & 0 & 0 \\
\hline 2 & & $a^{3} \mathrm{~F}_{3}$ & 951 & 988 & 972 \\
\hline 3 & & $a^{3} \mathrm{~F}_{2}$ & 1597 & 1676 & 1645 \\
\hline 4 & $3 \mathrm{~d}^{7} 4 \mathrm{~s}$ & $a^{5} \mathrm{~F}_{5}$ & 3351 & 2899 & 2876 \\
\hline 5 & & $a^{5} \mathrm{~F}_{4}$ & 4029 & 3562 & 3550 \\
\hline 6 & & $a^{5} \mathrm{~F}_{3}$ & 4561 & 4088 & 4082 \\
\hline 7 & & $a^{5} \mathrm{~F}_{2}$ & 4950 & 4476 & 4474 \\
\hline 8 & & $a^{5} \mathrm{~F}_{1}$ & 5205 & 4732 & 4731 \\
\hline 9 & $3 \mathrm{~d}^{7} 4 \mathrm{~s}$ & $b^{3} \mathrm{~F}_{4}$ & 9813 & 9642 & 9623 \\
\hline 10 & & $b^{3} \mathrm{~F}_{3}$ & 10709 & 10531 & 10523 \\
\hline 11 & & $b^{3} \mathrm{~F}_{2}$ & 11322 & 11156 & 11153 \\
\hline 12 & $3 d^{8}$ & $a^{1} \mathrm{D}_{2}$ & 11651 & 14367 & 14378 \\
\hline 13 & & $a^{3} \mathrm{P}_{2}$ & 13261 & 16022 & 15970 \\
\hline 14 & & $a^{3} \mathrm{P}_{1}$ & 13404 & 16094 & 16094 \\
\hline 15 & & $a^{3} \mathrm{P}_{0}$ & 13593 & 16305 & 16297 \\
\hline
\end{tabular}

$a^{5} \mathrm{~F}_{5}$, decays by a very weak magnetic dipole transition to the ground $a^{3} \mathrm{~F}_{4}$ level. We discuss this transition in more detail at the end of this section.

We find very close agreement with Nussbaumer \& Storey (1988) for transition probabilities within the $a^{3} \mathrm{~F}$ and $a^{5} \mathrm{~F}$ terms, with differences less than $1 \%$ between the two 
Table 5. Weighted $L S$ oscillator strengths, $g f$, in the length and velocity formulations from the energetically lowest three terms.

\begin{tabular}{|c|c|c|c|c|c|c|}
\hline \multicolumn{5}{|c|}{ Transition } & $g f_{L}$ & $g f_{V}$ \\
\hline \multirow[t]{3}{*}{$3 d^{8}$} & ${ }^{3} \mathrm{~F}$ & - & $3 d^{7} 4 p$ & ${ }^{3} \mathrm{G}^{\circ}$ & 0.17 & 0.21 \\
\hline & & - & & ${ }^{3} \mathrm{~F}^{\mathrm{o}}$ & 1.34 & 1.55 \\
\hline & & - & & ${ }^{3} \mathrm{D}^{\circ}$ & 0.75 & 0.81 \\
\hline \multirow[t]{3}{*}{$3 d^{7} 4 s$} & ${ }^{5} \mathrm{~F}$ & - & $3 d^{7} 4 p$ & ${ }^{5} \mathrm{~F}^{\mathrm{O}}$ & 10.75 & 10.70 \\
\hline & & - & & ${ }^{5} \mathrm{D}^{\mathrm{o}}$ & 7.30 & 6.62 \\
\hline & & - & & ${ }^{5} \mathrm{G}^{\circ}$ & 14.23 & 14.97 \\
\hline \multirow[t]{3}{*}{$3 d^{7} 4 s$} & ${ }^{3} \mathrm{~F}$ & - & $3 \mathrm{~d}^{7} 4 \mathrm{p}$ & ${ }^{3} \mathrm{G}^{\mathrm{o}}$ & 7.51 & 9.09 \\
\hline & & - & & ${ }^{3} \mathrm{~F}^{\mathrm{o}}$ & 5.82 & 6.52 \\
\hline & & - & & ${ }^{3} \mathrm{D}^{\circ}$ & 4.29 & 4.42 \\
\hline
\end{tabular}

calculations. Differences of up to $45 \%$ occur for transitions between the $b^{3} \mathrm{~F}$ and two lower terms and within that term.

We also find reasonable agreement with the work of Quinet (1998) for the transitions of interest here, the magnetic dipole transition probabilities within the $a^{3} \mathrm{~F}$ and $a^{5} \mathrm{~F}$ terms. His results differ by no more than $20 \%$ from the present work for those transitions. Larger differences, of up to $60 \%$ are seen for transitions from the higher $b^{3} \mathrm{~F}, a^{1} \mathrm{D}$ and $a^{3} \mathrm{P}$ terms to the ground $a^{3} \mathrm{~F}$ term, although the remaining transitions show much smaller differences, typically about 10\%. The calculation of Quinet (1998) uses a similar configuration basis to the present work but employs a HartreeFock approach which incorporates fitting of Slater parameters to experimental energies. Consequently, that method yields calculated energies in much better agreement with experiment than our $a b$ initio results. We do, however, make empirical corrections to the $L S$-Hamiltonian matrix to bring our final calculated level energies into good agreement with experiment, to ensure that the spin-orbit interactions between levels of different terms are corrected for any errors due to incorrect term energy separations. We also use experimental energies in the calculation of transition probabilities from line strengths.

Table 6 also shows the forbidden transition probabilities calculated by Raassen et al (1998), which show very good agreement with the present work, differing by less than $10 \%$ in all but three cases and agreeing within $1 \%$ for all the magnetic dipole transitions between the levels of the $a^{3} \mathrm{~F}$ and $a^{5} \mathrm{~F}$ terms. Despite this very close agreement for the infrared transitions, the larger differences between our results and those of Quinet (1998) should probably be viewed as a measure of the uncertainty in the values of the transition probabilities for this rather complex ion.

The $a^{5} \mathrm{~F}_{5}-a^{3} \mathrm{~F}_{4}$, which was briefly referred to above, requires a separate discussion. Since there are no magnetic dipole matrix elements between different terms, this transition must proceed via an interaction between $3 \mathrm{~d}^{8} a^{5} \mathrm{~F}_{4}$ and $3 \mathrm{~d}^{7} 4 \mathrm{~s} a^{3} \mathrm{~F}_{4}$. As discussed by Nussbaumer \& Storey (1988), there is no direct spin-orbit interaction between $a^{3} \mathrm{~F}_{4}$ and $a^{5} \mathrm{~F}_{4}$ levels; only very small two-body fine-structure terms. A combination of configuration interaction between $a^{3} \mathrm{~F}$ and $b^{3} \mathrm{~F}$, plus spin-orbit interaction between $b^{3} \mathrm{~F}_{4}$ and $a^{5} \mathrm{~F}_{4}$, is also excluded because the matrix element for the $a^{3} \mathrm{~F}-b^{3} \mathrm{~F}$ electrostatic interaction is also zero. In practice, the transition can occur via configuration interaction between $a^{3} \mathrm{~F}$ and $c^{3} \mathrm{~F}$, which is non-zero, and spin-orbit interaction between $c^{3} \mathrm{~F}_{4}$ and $a^{5} \mathrm{~F}_{4}$, but the $c^{3} \mathrm{~F}$ is at relatively high energy $\left(40228 \mathrm{~cm}^{-1}\right)$ so the interaction is very weak.

In their discussion of this transition, Nussbaumer \&
Storey (1988) point out that since it is very weak, it might be significantly altered if the relativistic corrections to the magnetic dipole operator described by Eissner \& Zeippen (1981) were included. These corrections are included in the current work and we find that they change the $a^{5} \mathrm{~F}_{5}-a^{3} \mathrm{~F}_{4}$ transition probability by less than $1 \%$. Among the four lowest terms, the largest changes due to these corrections, ranging from 0 to $3.2 \%$, are seen in the $b^{3} \mathrm{~F}-a^{5} \mathrm{~F}$ multiplet. We also find that the exclusion of two-body fine-structure terms only changes the $a^{5} \mathrm{~F}_{5}-a^{3} \mathrm{~F}_{4}$ transition probability by a small amount (5\%).

For this transition, our calculated probability of $9.93 \times 10^{-6} \mathrm{~s}^{-1}$ is a factor 2.0 times larger than the value of Nussbaumer \& Storey (1988) and a factor 3.2 times larger than that found by Quinet (1998). Raassen et al (1998) do not give a value for this transition probability. In addition to the mechanism described above, we find that the $a^{3} \mathrm{~F}_{4}$ and $a^{5} \mathrm{~F}_{4}$ levels can interact via ${ }^{3} \mathrm{~F}$ terms belonging to the $3 \mathrm{~d}^{7} 4 \mathrm{~d}$ configuration. We believe that it is presence of these interactions that causes the large differences between the different calculations. The present calculation and that of Nussbaumer \& Storey (1988) utilise a short-range correlation $4 \mathrm{~d}$ orbital to approximate the effect of the infinite series of bound and continuum d orbitals that should be accounted for. Quinet (1998) does not use such orbitals and so underestimates the contribution of the $3 \mathrm{~d}^{7} \mathrm{nd}$ configurations to the transition probability.

Within the $a^{3} \mathrm{~F}$ and $a^{5} \mathrm{~F}$ terms, radiative decays occur stepwise between adjacent levels with probabilities of order $10^{-2} \mathrm{~s}^{-1}$, while the probability of a transition between the $a^{5} \mathrm{~F}$ and $a^{3} \mathrm{~F}$ terms is typically four orders of magnitude smaller. Hence the only transition of significance between the two terms is $a^{5} \mathrm{~F}_{5}-a^{3} \mathrm{~F}_{4}$. In a physical situation where levels are excited by electron collisions and decay by radiative decay or collisional de-excitation this transition alone determines the critical electron density above which the rate of collisional de-excitation of the $a^{5} \mathrm{~F}$ levels is larger than radiative decay. We return to this point in Section 4.1 .

\section{SCATTERING CALCULATIONS}

The Breit-Pauli R-matrix method which is used in this calculation is described fully elsewhere (Hummer et al 1993 Berrington et al 1995) and references therein. The calculations described here were made with the parallel versions of the codes ${ }^{2}$ We use an R-matrix boundary radius of $14.9 \mathrm{au}$, to encompass the most extended target orbital (4p). The expansion of each scattered electron partial wave is over a basis of 12 functions within the R-matrix boundary, and the partial wave expansion extends to a maximum of $2 J=19$.

Collision strengths are computed at 20000 equally spaced values of the energy in the resonance region and a further 1000 values in the region where all scattering channels are open, up to an incident electron energy of 1.5 Rydberg. In Figure 3 we illustrate our results with the calculated collision strengths between the three levels of the ground $3 \mathrm{~d}^{8}{ }^{3} \mathrm{~F}$ term as a function of final electron energy up to 0.4 Rydberg

2 See Badnell: R-matrix write-up on WWW. URL: http://amdpp.phys.strath.ac.uk/tamoc/codes/parallel/PWRITEUP. 

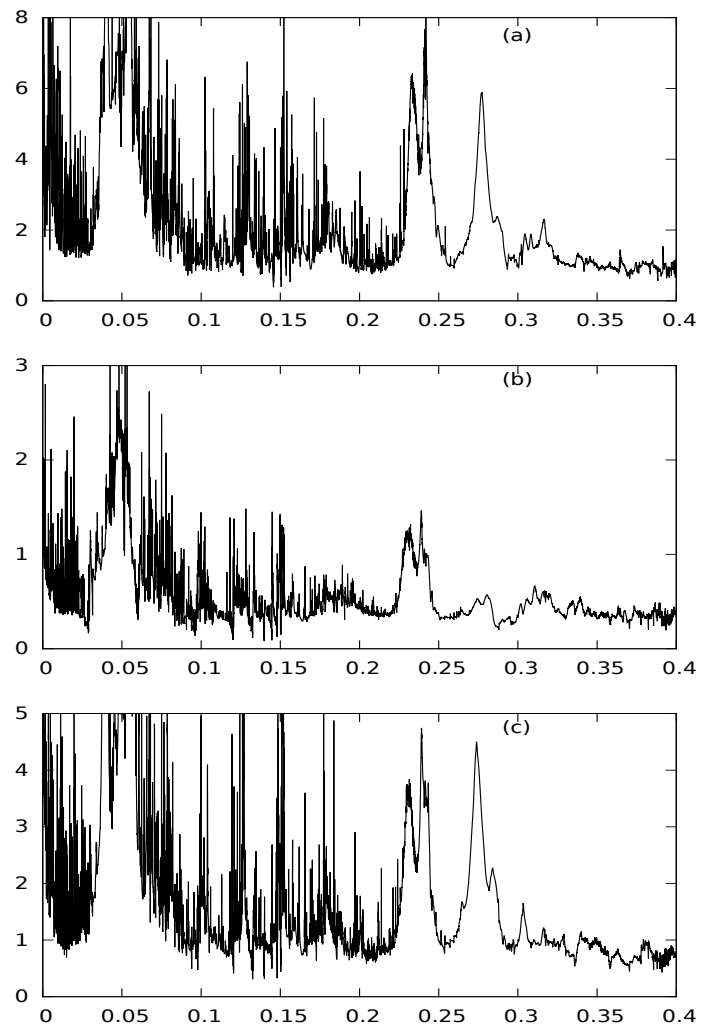

Figure 3. Collision strength (vertical axis) versus final electron energy in Rydberg (horizontal axis) for the (a) 1-2, (b) 1-3 and (c) 2-3 transitions. For level indexing refer to Table 4

above threshold. Due to the multiple close lying thresholds the collision strengths display very dense and complex resonance structures.

\section{RESULTS AND DISCUSSION}

In Table 7, the final thermally averaged collision strengths between the 15 energetically lowest levels are given as a function of electron temperature. In the energy region where all scattering channels are open we find some small irregular features in the collision strengths that are almost certainly non-physical and caused by the correlation orbitals in the target representation. We have computed thermally averaged collision strengths for the transitions and temperature range given in Table 7 both including and excluding the contribution from the region of all channels open, and find the largest change for any transition is $0.3 \%$ at $\log _{10} T=4.0$, $2.4 \%$ at $\log _{10} T=4.2$ and $9.4 \%$ at $\log _{10} T=4.4$. For the excitations from the $a^{3} \mathrm{~F}$ levels to the $a^{5} \mathrm{~F}$ levels, the maximum difference at any temperature is $2.2 \%$. Hence for the infrared transitions of interest here the contribution from any non-physical features is insignificant. The values tabulated in Table 7 were computed using the full energy range.

\subsection{The IR line ratios}

The transitions between the levels of the $a^{3} \mathrm{~F}$ and $a^{5} \mathrm{~F}$ terms give rise to mid-infrared lines lying between 10.5 and $39.3 \mu \mathrm{m}$. The relative intensity of the two lines arising from

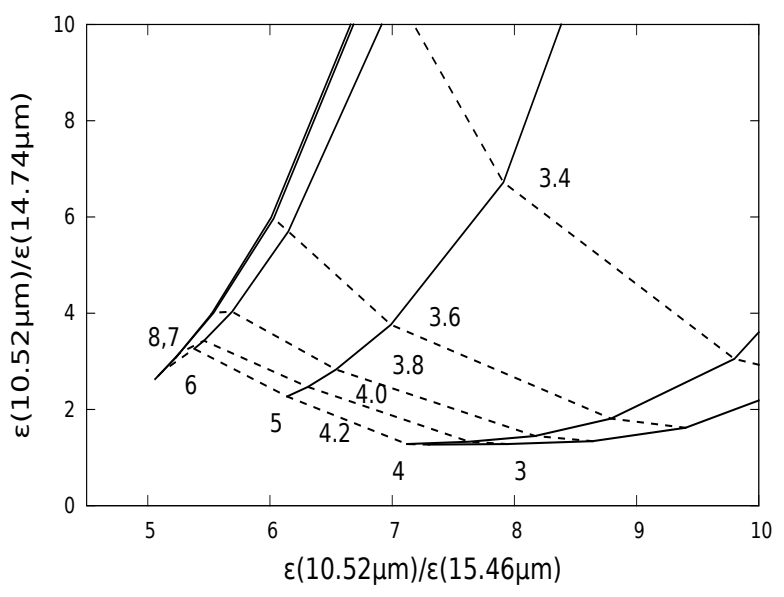

Figure 4. Emissivity ratios of pairs of Co II infrared lines, showing contours of constant electron temperature (dashed lines) and electron number density (solid lines). Lines are labelled with the $\log _{10}$ of temperature in $\mathrm{K}$ or density in $\mathrm{cm}^{-3}$.

the $a^{3} \mathrm{~F}$ term $(10.52 \mu \mathrm{m}$ and $15.46 \mu \mathrm{m}$, see Figure 2$)$ is sensitive to electron density for densities less than about $10^{6} \mathrm{~cm}^{-3}$ and relatively insensitive to temperature for temperatures greater than about $3000 \mathrm{~K}$ due to the low excitation energies. The lines from within the higher lying $a^{5} \mathrm{~F}$ term, the $14.74 \mu \mathrm{m}$ line for example, are more sensitive to electron temperature. Ratios of the intensities of these three lines can therefore be used to simultaneously determine the electron temperature and density of the emitting material if they lie in the appropriate range. This is illustrated in Figure 4 where we plot the $10.52 / 14.74 \mu \mathrm{m}$ ratio against the $10.52 / 15.46 \mu \mathrm{m}$ ratio for various temperatures and densities. We discussed in Section 2.2 the role of the $a^{5} \mathrm{~F}_{5}-a^{3} \mathrm{~F}_{4}$ transition in determining the relative populations of the $a^{5} \mathrm{~F}$ and $a^{3} \mathrm{~F}$ levels and the rather large differences in the calculated values for the radiative decay probability for this transition. The value calculated by Quinet (1998) is about a factor of three smaller than ours and a lower transition probability would mean that the critical density for collisional de-excitation of the $a^{5} \mathrm{~F}$ levels would be three times smaller. Above the critical density the relative level populations tend to their Boltzmann values and depend only on temperature as shown by the lines in Figure 2 corresponding to electron number densities of $10^{7}$ and $10^{8} \mathrm{~cm}^{-3}$. If the transition probability of Quinet (1998) were adopted, these high density limit values of the ratios would be reached at a density three times lower.

\section{CONCLUSIONS}

In this paper, the Co II forbidden transitions between the fifteen lowest energy levels of singly-ionised cobalt, $\mathrm{Co}^{+}$, have been investigated. Radiative transition probabilities and, for the first time for this ion, collision strengths for excitation by electrons and their thermally-averaged values based on a Maxwell-Boltzmann electron energy distribution have been calculated. An elaborate atomic target was used to perform the scattering calculations using the R-matrix method in the Breit-Pauli approximation and intermediate coupling scheme. 
We have compared the radiative transition probabilities with those from three previous calculations by Nussbaumer \& Storey (1988), Quinet (1998) and Raassen et al (1998), and briefly discussed the consequences of the differences seen for the spectroscopy of the infrared lines. Although there is good agreement between our data and the previous works, there are also some significant differences, particularly for the important $a^{5} \mathrm{~F}_{5}-a^{3} \mathrm{~F}_{4}$ transition. This probability is clearly very sensitive to the choice of configurations and orbitals, and we have argued that our basis and orbitals give the most reliable value for this probability.

The diagnostic potential of the infrared lines is illustrated by showing that the three lines at 10.52, 14.74 and $15.46 \mu \mathrm{m}$ can potentially be used to measure the electron density and temperature of the emitting region. These lines are of particular use in the study of supernovae.

\section{ACKNOWLEDGMENTS}

PJS and CJZ acknowledge financial support from the Atomic Physics for Astrophysics Project (APAP) funded by the Science and Technology Facilities Council (STFC). The present collaboration benefited from visits to Meudon by PJS from 1998 to 2013, with support provided by the Observatoire de Paris and by the Université Paris 7. The hospitality of the Observatoire de Paris was much appreciated.

\section{REFERENCES}

Adelman S.J., Gulliver A.F., Lodén, L.O., 2000, A\&A, 353, 335

Axelrod T.S., 1980, PhD thesis (UCRL-52994), University of California Santa Cruz

Badnell, N.R., 2011, Comput. Phys. Commun. 182, 1528

Baluteau J.P., Zavagno A., Morisset C., Péquignot D., 1995, A\&A, 303, 175

Bergemann M., Pickering J.C., Gehren T., 2010, MNRAS 401, 1334

Berrington K.A., Burke P.G., Chang J.J., Chivers A.T., Robb W.D., Taylor K.T., 1974, Comp. Phys. Comm., 8, 149

Berrington K.A., Burke P.G., Butler K., Seaton M.J., Storey P.J., Taylor K.T., Yu Yan., 1987, J. Phys. B, 20, 6379

Berrington K.A., Eissner W.B., Norrington P.H., 1995, Comp. Phys. Comm., 92, 290

Bowers E.J.C., Meikle W.P.S., Geballe T.R. et al, 1997, MNRAS, 290, 663

Churazov E., Sunyaev R., Isern J. et al, 2014, Nature, 512, 406

Colgate S.A., McKee C., 1969, ApJ, 157, 623

Crespo López-Urrutia J.R., Ulbe M., Neger T., Jäger H., 1994, J. Quant. Spect. Rad. Trans., 52, 89

Dinerstein H.L., 1995, "Infrared emission lines as probes of gaseous nebulae", in: "The Analysis of Emission Lines", eds. Williams R.E. and Livio M., Space Telescope Science Institute Symposium Series 8

Eissner W., Jones M. \& Nussbaumer H., 1974, Comput. Phys. Commun. 8, 270
Eissner, W., Zeippen, C.J., 1981, J. Phys. B. 14, 2125

Esteban C., Peimbert M., García-Rojas J., Ruiz M.T., Peimbert A., Rodríguez M., 2004, MNRAS, 355, 229

Fang X., Liu X.-W., 2011, MNRAS, 415, 181

Hummer D.G., Berrington K.A., Eissner W., Pradhan A.K., Saraph H.E., Tully J.A., 1993, A\&A, 279, 298

Jennings D.E., Boyle R.J., Wiedemann G.R., Moseley S.H., 1993, ApJ, 408, 277

Jerkstrand A., Fransson C., Maguire K., Smartt S., Ergon M., Spyromilio J., 2012, A\&A, 546, A28

Kerola D.X., Aller L.H., 1976, Publications of the Astronomical Society of the Pacific, 88, 122

Kuchner M.J., Kirshner R.P., Pinto P.A., Leibundgut B., 1994, ApJ, 426, L89

Li H., McCray R., Sunyaev R.A., 1993, ApJ, 419, 824

Liu W., Jeffery D.J., Schultz D.R., Quinet P., Shaw J., Pindzola M.S., 1997, ApJ, 489, L141

McKenna F.C., Keenan F.P., Hambly N.C. et al, 1997, ApJS, 109, 225

Mullman K.L., Cooper J.C., Lawler J.E., 1998, ApJ, 495, 503

Mullman K.L., Lawler J.E., Zsargó J., Federman S.R., 1998, ApJ, 500, 1064

Nussbaumer H., Storey P.J., 1978, A\&A, 64, 139

Nussbaumer H, Storey P.J., 1988, A\&A, 200, L25

del Peloso E.F., Cunha K., da Silva L., Porto de Mello G.F., 2005, A\&A, 441, 1149

Pickering J.C., Raassen A.J.J., Uylings P.H.M., Johansson S., 1998, ApJS, 117, 261

Pottasch S.R., Surendiranath R., 2005, A\&A, 444, 861

Quinet P., 1998, A\&AS, 129, 147

Raassen A.J.J., Pickering J.C., Uylings P.H.M., 1998, A\&AS 130, 541

Ruffon M.P., Pickering J.C., 2013, ApJS, 207, 20

Sahu D.K., Anupama G.C., Srividya S., Muneer S., 2006, MNRAS, 372, 1315

Salih S., Lawler J.E., Whaling W., 1985, Phys. Rev. A, 31, 744

Sharpee B., Williams R., Baldwin J.A., van Hoof P.A.M., 2003, ApJS, 149, 157

Sharpee B., Zhang Y., Williams R., Pellegrini E., Cavagnolo K., Baldwin J.A., Phillips M., X.-W. Liu, 2007, ApJ, 659, 1265

Sugar J., Corliss C., 1981, J. Phys. Chem. Ref. Data, 10, 1097

Sugar J., Corliss C., 1985, J. Phys. Chem. Ref. Data, 14, 1 Wang W., Liu X.-W., 2007, MNRAS, 381, 669

Zhang Y., Liu X.-W., Luo S.-G., Péquignot D., Barlow M.J., 2005, A\&A, 442, 249 
Table 6. Transition probabilities in $\mathrm{s}^{-1}$ among the energetically lowest 15 levels (see Table 4 for level indexing) as obtained from the current work (CW), Nussbaumer \& Storey (1988) (NS), Quinet (1998) (Q) and Raassen et al (1998) (RPU) where $i$ and $j$ refer to the lower and upper levels respectively. Only transition probabilities that are at least $1 \%$ of the total probability from a given upper level are listed. The powers of 10 by which the number is to be multiplied are given in brackets.

\begin{tabular}{|c|c|c|c|c|c|c|c|c|c|c|c|}
\hline \multicolumn{2}{|c|}{ Transition } & CW & NS & $\mathrm{Q}$ & $\mathrm{RPU}$ & & Transition & CW & NS & $\mathrm{Q}$ & $\mathrm{RPU}$ \\
\hline$j$ & $i$ & & & & & & $j$ & $i$ & & & \\
\hline 2 & 1 & $2.23(-2)$ & $2.23(-2)$ & $2.21(-2)$ & $2.24(-2)$ & 10 & 9 & $1.88(-2)$ & $1.87(-2)$ & $1.67(-2)$ & $1.88(-2)$ \\
\hline 3 & 2 & $9.71(-3)$ & $9.73(-3)$ & $9.88(-3)$ & $9.73(-3)$ & 11 & 2 & $2.73(-2)$ & $1.87(-2)$ & $3.16(-2)$ & $1.72(-2)$ \\
\hline 4 & 1 & $9.13(-6)$ & $4.90(-6)$ & $3.09(-6)$ & - & 11 & 3 & $2.81(-2)$ & $3.09(-2)$ & $4.06(-2)$ & $2.57(-2)$ \\
\hline 5 & 4 & $1.24(-2)$ & $1.23(-2)$ & $1.03(-2)$ & $1.24(-2)$ & 11 & 7 & $4.61(-3)$ & $5.30(-3)$ & $4.34(-3)$ & $4.39(-3)$ \\
\hline 6 & 5 & $1.08(-2)$ & $1.08(-2)$ & $9.27(-3)$ & $1.09(-2)$ & 11 & 8 & $1.41(-2)$ & $1.69(-2)$ & $1.34(-2)$ & $1.35(-2)$ \\
\hline 7 & 6 & $5.72(-3)$ & $5.72(-3)$ & $5.00(-3)$ & $5.74(-3)$ & 11 & 10 & $7.82(-3)$ & $8.32(-3)$ & $7.26(-3)$ & $8.19(-3)$ \\
\hline 8 & 3 & $4.33(-5)$ & - & - & - & 12 & 1 & $7.61(-3)$ & - & $9.74(-3)$ & - \\
\hline 8 & 7 & $1.78(-3)$ & $1.78(-3)$ & $1.57(-3)$ & $1.79(-3)$ & 12 & 2 & $1.67(-1)$ & - & $1.74(-1)$ & - \\
\hline 9 & 1 & $3.36(-2)$ & $3.85(-2)$ & $5.36(-2)$ & $3.43(-2)$ & 12 & 3 & $7.99(-2)$ & - & $8.16(-2)$ & $1.27(-3)$ \\
\hline 9 & 2 & $3.98(-3)$ & $4.91(-3)$ & $6.33(-3)$ & $4.01(-3)$ & 13 & 1 & $3.34(-2)$ & - & $4.98(-2)$ & - \\
\hline 9 & 4 & $2.90(-2)$ & $2.89(-2)$ & $2.66(-2)$ & $2.81(-2)$ & 13 & 2 & $6.98(-2)$ & - & $6.70(-2)$ & - \\
\hline 9 & 5 & $3.27(-3)$ & $2.82(-3)$ & $2.98(-3)$ & $3.07(-3)$ & 13 & 3 & $1.82(-2)$ & - & $1.63(-2)$ & - \\
\hline 9 & 6 & $5.21(-3)$ & $4.28(-3)$ & $4.81(-3)$ & $5.05(-3)$ & 13 & 12 & $3.01(-2)$ & - & $2.96(-2)$ & - \\
\hline 10 & 1 & $1.45(-2)$ & $1.63(-3)$ & $2.30(-2)$ & $1.51(-2)$ & 14 & 2 & $2.92(-2)$ & - & $4.20(-2)$ & - \\
\hline 10 & 2 & $2.22(-2)$ & $2.58(-2)$ & $3.47(-2)$ & $2.25(-2)$ & 14 & 3 & $1.19(-2)$ & - & $1.68(-2)$ & - \\
\hline 10 & 3 & $5.60(-3)$ & $6.97(-3)$ & $8.74(-3)$ & $5.65(-3)$ & 14 & 11 & $1.99(-3)$ & - & - & - \\
\hline 10 & 5 & $4.43(-3)$ & $6.41(-3)$ & $4.17(-3)$ & $3.97(-3)$ & 14 & 12 & $2.78(-2)$ & - & $2.55(-2)$ & - \\
\hline 10 & 6 & $4.95(-3)$ & $4.91(-3)$ & $4.53(-3)$ & $4.57(-3)$ & 15 & 3 & $3.70(-2)$ & - & $5.34(-2)$ & - \\
\hline 10 & 7 & $1.04(-2)$ & $1.04(-2)$ & $9.59(-3)$ & $9.65(-3)$ & & & & & & \\
\hline
\end{tabular}


Table 7: Thermally-averaged collision strengths among the 15 energetically lowest levels as a function of log 10 of temperature in $\mathrm{K}$ where $i$ and $j$ refer to the index of the lower and upper level respectively (see Table 4 for indexing).

\begin{tabular}{|c|c|c|c|c|c|c|c|c|c|c|c|c|c|c|}
\hline \multirow[t]{2}{*}{$i$} & \multirow[t]{2}{*}{$j$} & \multicolumn{13}{|c|}{$\log _{10} T$} \\
\hline & & 2.0 & 2.2 & 2.4 & 2.6 & 2.8 & 3.0 & 3.2 & 3.4 & 3.6 & 3.8 & 4.0 & 4.2 & 4.4 \\
\hline 1 & 3 & 1.449 & 1.320 & 1.189 & 1.064 & 0.947 & 0.845 & 0.781 & 0.775 & 0.802 & 0.807 & 0.772 & 0.709 & 0.636 \\
\hline 1 & 5 & 2.292 & 2.136 & 1.988 & 1.918 & 1.978 & 2.138 & 2.285 & 2.314 & 2.191 & 1.955 & 1.684 & 1.447 & 1.251 \\
\hline 1 & 6 & 0.674 & 0.751 & 0.834 & 0.937 & 1.054 & 1.149 & 1.186 & 1.150 & 1.049 & 0.909 & 0.767 & 0.656 & 0.572 \\
\hline 1 & 7 & 0.235 & 0.241 & 0.271 & 0.319 & 0.366 & 0.395 & 0.401 & 0.385 & 0.349 & 0.301 & 0.254 & 0.220 & 0.196 \\
\hline 1 & 8 & 0.037 & 0.039 & 0.046 & 0.057 & 0.071 & 0.081 & 0.084 & 0.081 & 0.073 & 0.063 & 0.053 & 0.047 & 0.043 \\
\hline 1 & 11 & 0.143 & 0.146 & 0.150 & 0.156 & 0.161 & 0.161 & 0.155 & 0.146 & 0.136 & 0.127 & 0.128 & 0.139 & 0.149 \\
\hline 1 & 12 & 0.679 & 0.726 & 0.809 & 0.926 & 1.033 & 1.084 & 1.080 & 1.055 & 1.029 & 1.003 & 0.973 & 0.942 & 0.915 \\
\hline 1 & 13 & 0.641 & 0.654 & 0.654 & 0.637 & 0.618 & 0.620 & 0.642 & 0.672 & 0.706 & 0.741 & 0.769 & 0.783 & 0.773 \\
\hline 1 & 14 & 0.253 & 0.251 & 0.241 & 0.228 & 0.218 & 0.216 & 0.220 & 0.228 & 0.243 & 0.268 & 0.297 & 0.320 & 0.328 \\
\hline 1 & 15 & 0.074 & 0.070 & 0.065 & 0.062 & 0.061 & 0.063 & 0.066 & 0.069 & 0.072 & 0.077 & 0.086 & 0.093 & 0.095 \\
\hline 2 & 3 & 4.806 & 4.372 & 3.886 & 3.409 & 2.967 & 2.590 & 2.361 & 2.357 & 2.475 & 2.513 & 2.399 & 2.182 & 1.919 \\
\hline 2 & 8 & 0.258 & 0.270 & 0.296 & 0.340 & 0.390 & 0.431 & 0.453 & 0.452 & 0.426 & 0.381 & 0.331 & 0.290 & 0.257 \\
\hline 2 & 9 & 0.733 & 0.795 & 0.835 & 0.847 & 0.836 & 0.810 & 0.778 & 0.743 & 0.708 & 0.674 & 0.654 & 0.654 & 0.649 \\
\hline 2 & 10 & 1.314 & 1.334 & 1.317 & 1.273 & 1.225 & 1.182 & 1.137 & 1.086 & 1.031 & 0.979 & 0.957 & 0.980 & 1.006 \\
\hline 2 & 11 & 0.486 & 0.484 & 0.490 & 0.509 & 0.531 & 0.545 & 0.544 & 0.531 & 0.512 & 0.495 & 0.495 & 0.518 & 0.540 \\
\hline 2 & 12 & 0.448 & 0.455 & 0.497 & 0.579 & 0.672 & 0.732 & 0.749 & 0.740 & 0.723 & 0.700 & 0.676 & 0.649 & 0.617 \\
\hline 2 & 13 & 0.550 & 0.554 & 0.551 & 0.544 & 0.538 & 0.544 & 0.562 & 0.581 & 0.596 & 0.607 & 0.616 & 0.619 & 0.610 \\
\hline 2 & 14 & 0.370 & 0.397 & 0.409 & 0.406 & 0.397 & 0.391 & 0.389 & 0.388 & 0.392 & 0.402 & 0.418 & 0.433 & 0.436 \\
\hline 2 & 15 & 0.070 & 0.064 & 0.058 & 0.054 & 0.053 & 0.055 & 0.059 & 0.065 & 0.073 & 0.084 & 0.094 & 0.100 & 0.103 \\
\hline 3 & 4 & 0.173 & 0.166 & 0.158 & 0.149 & 0.141 & 0.137 & 0.139 & 0.139 & 0.132 & 0.118 & 0.101 & 0.086 & 0.075 \\
\hline 3 & 5 & 0.783 & 0.771 & 0.747 & 0.712 & 0.673 & 0.642 & 0.623 & 0.606 & 0.574 & 0.520 & 0.455 & 0.394 & 0.341 \\
\hline 3 & 6 & 1.197 & 1.183 & 1.151 & 1.100 & 1.051 & 1.028 & 1.030 & 1.030 & 0.992 & 0.908 & 0.801 & 0.698 & 0.606 \\
\hline 3 & 7 & 1.208 & 1.174 & 1.121 & 1.072 & 1.054 & 1.077 & 1.117 & 1.131 & 1.090 & 0.995 & 0.876 & 0.765 & 0.669 \\
\hline 4 & 6 & 0.395 & 0.419 & 0.485 & 0.581 & 0.666 & 0.703 & 0.686 & 0.630 & 0.549 & 0.469 & 0.440 & 0.499 & 0.594 \\
\hline 4 & 7 & 0.127 & 0.156 & 0.196 & 0.239 & 0.274 & 0.285 & 0.271 & 0.240 & 0.202 & 0.167 & 0.150 & 0.162 & 0.187 \\
\hline 4 & 8 & 0.073 & 0.075 & 0.082 & 0.095 & 0.105 & 0.104 & 0.093 & 0.077 & 0.061 & 0.049 & 0.042 & 0.045 & 0.051 \\
\hline 4 & 9 & 18.971 & 18.883 & 18.780 & 18.640 & 18.447 & 18.175 & 17.721 & 16.898 & 15.540 & 13.714 & 11.799 & 10.115 & 8.614 \\
\hline 4 & 10 & 0.276 & 0.240 & 0.211 & 0.190 & 0.174 & 0.161 & 0.150 & 0.145 & 0.145 & 0.157 & 0.205 & 0.294 & 0.375 \\
\hline 4 & 11 & 0.044 & 0.052 & 0.056 & 0.053 & 0.047 & 0.041 & 0.036 & 0.034 & 0.034 & 0.036 & 0.046 & 0.066 & 0.085 \\
\hline 4 & 12 & 0.298 & 0.297 & 0.301 & 0.304 & 0.303 & 0.297 & 0.286 & 0.272 & 0.258 & 0.244 & 0.230 & 0.218 & 0.201 \\
\hline 4 & 13 & 0.556 & 0.548 & 0.549 & 0.554 & 0.558 & 0.558 & 0.553 & 0.545 & 0.534 & 0.521 & 0.508 & 0.494 & 0.468 \\
\hline 4 & 14 & 0.017 & 0.021 & 0.025 & 0.027 & 0.027 & 0.027 & 0.025 & 0.024 & 0.023 & 0.023 & 0.027 & 0.033 & 0.037 \\
\hline 4 & 15 & 0.036 & 0.029 & 0.023 & 0.018 & 0.014 & 0.011 & 0.010 & 0.009 & 0.008 & 0.007 & 0.008 & 0.009 & 0.009 \\
\hline 5 & 6 & 7.160 & 7.608 & 8.160 & 8.611 & 8.810 & 8.785 & 8.694 & 8.618 & 8.446 & 8.035 & 7.446 & 6.867 & 6.285 \\
\hline 5 & 7 & 0.499 & 0.491 & 0.506 & 0.548 & 0.587 & 0.596 & 0.570 & 0.521 & 0.456 & 0.391 & 0.368 & 0.419 & 0.499 \\
\hline 5 & 8 & 0.138 & 0.168 & 0.206 & 0.240 & 0.256 & 0.251 & 0.232 & 0.205 & 0.175 & 0.148 & 0.136 & 0.151 & 0.178 \\
\hline 5 & 9 & 8.909 & 8.860 & 8.811 & 8.728 & 8.556 & 8.267 & 7.865 & 7.337 & 6.647 & 5.831 & 5.067 & 4.493 & 4.005 \\
\hline 5 & 10 & 8.662 & 8.640 & 8.655 & 8.684 & 8.690 & 8.634 & 8.451 & 8.055 & 7.388 & 6.496 & 5.563 & 4.743 & 4.022 \\
\hline 5 & 11 & 0.108 & 0.110 & 0.110 & 0.106 & 0.099 & 0.090 & 0.083 & 0.080 & 0.080 & 0.087 & 0.117 & 0.173 & 0.226 \\
\hline 5 & 12 & 0.262 & 0.251 & 0.243 & 0.234 & 0.226 & 0.216 & 0.207 & 0.197 & 0.187 & 0.177 & 0.168 & 0.161 & 0.150 \\
\hline 5 & 13 & 0.357 & 0.346 & 0.338 & 0.332 & 0.327 & 0.323 & 0.318 & 0.312 & 0.303 & 0.296 & 0.296 & 0.301 & 0.296 \\
\hline 5 & 14 & 0.275 & 0.276 & 0.277 & 0.275 & 0.273 & 0.270 & 0.267 & 0.262 & 0.255 & 0.248 & 0.242 & 0.239 & 0.229 \\
\hline 5 & 15 & 0.020 & 0.016 & 0.013 & 0.010 & 0.009 & 0.009 & 0.009 & 0.009 & 0.008 & 0.009 & 0.010 & 0.012 & 0.013 \\
\hline 6 & 7 & 5.975 & 6.146 & 6.457 & 6.876 & 7.209 & 7.337 & 7.361 & 7.372 & 7.285 & 6.978 & 6.489 & 5.970 & 5.433 \\
\hline 6 & 8 & 0.360 & 0.382 & 0.423 & 0.466 & 0.489 & 0.481 & 0.449 & 0.403 & 0.350 & 0.300 & 0.283 & 0.323 & 0.386 \\
\hline
\end{tabular}


Table 7: continued.

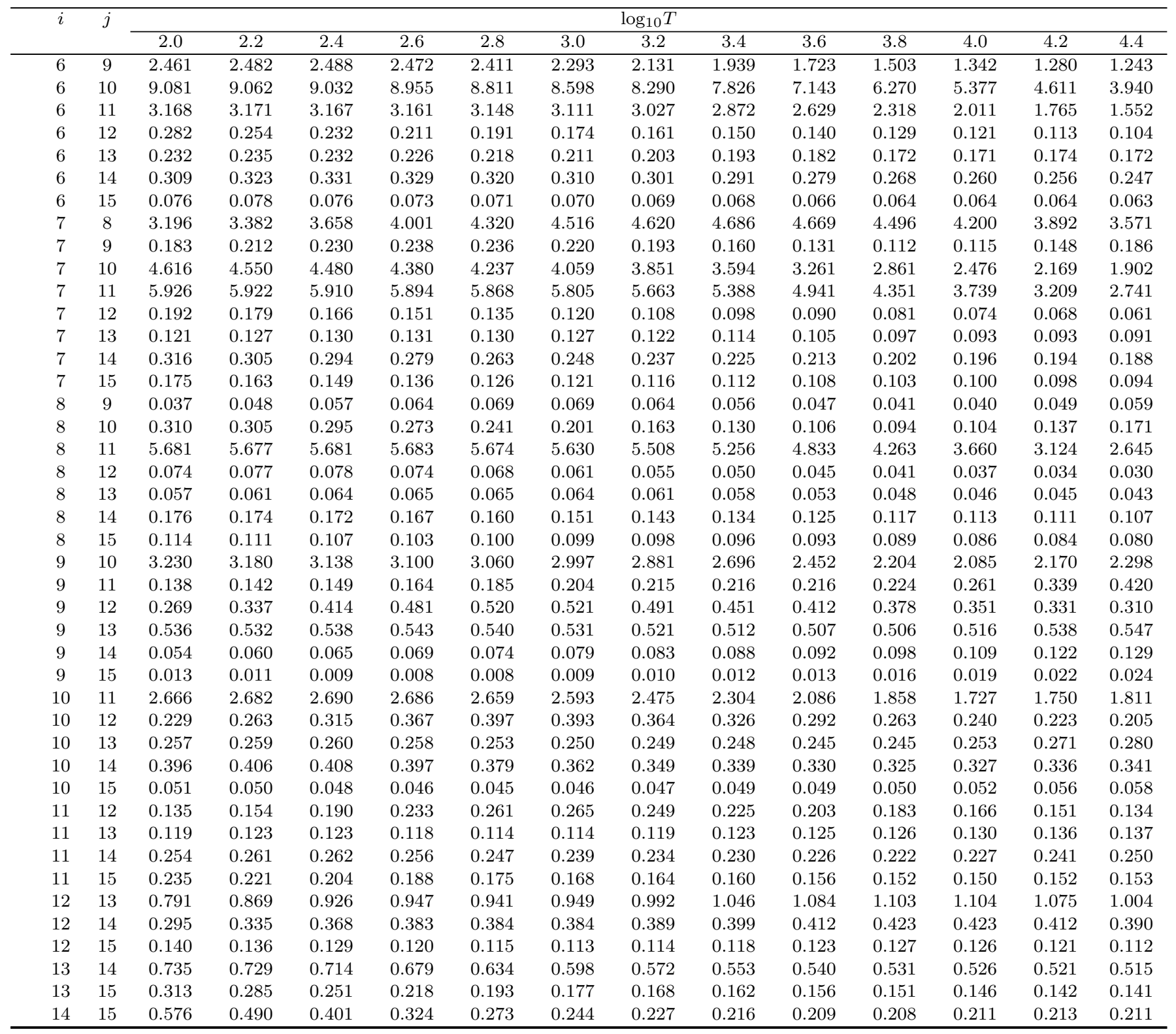

